\title{
iPhos: a toolkit to streamline the alkaline phosphatase-assisted comprehensive LC-MS phosphoproteome investigation
}

Tzu-Hsien Yang ${ }^{1}$, Hong-Tsun Chang ${ }^{1}$, Eric SL Hsiao², Juo-Ling Sun², Chung-Ching Wang ${ }^{1}$, Hsin-Yi Wu ${ }^{3}$, Pao-Chi Liao ${ }^{2 *}$, Wei-Sheng $\mathrm{Wu}^{1 *}$

From Asia Pacific Bioinformatics Network (APBioNet) Thirteenth International Conference on Bioinformatics (InCoB2014)

Sydney, Australia. 31 July - 2 August 2014

\begin{abstract}
Background: Comprehensive characterization of the phosphoproteome in living cells is critical in signal transduction research. But the low abundance of phosphopeptides among the total proteome in cells remains an obstacle in mass spectrometry-based proteomic analysis. To provide a solution, an alternative analytic strategy to confidently identify phosphorylated peptides by using the alkaline phosphatase (AP) treatment combined with high-resolution mass spectrometry was provided. While the process is applicable, the key integration along the pipeline was mostly done by tedious manual work.

Results: We developed a software toolkit, iPhos, to facilitate and streamline the work-flow of AP-assisted phosphoproteome characterization. The iPhos tookit includes one assister and three modules. The iPhos Peak Extraction Assister automates the batch mode peak extraction for multiple liquid chromatography mass spectrometry (LC-MS) runs. iPhos Module-1 can process the peak lists extracted from the LC-MS analyses derived from the original and dephosphorylated samples to mine out potential phosphorylated peptide signals based on mass shift caused by the loss of some multiples of phosphate groups. And iPhos Module-2 provides customized inclusion lists with peak retention time windows for subsequent targeted LC-MS/MS experiments. Finally, iPhos Module-3 facilitates to link the peptide identifications from protein search engines to the quantification results from pattern-based label-free quantification tools. We further demonstrated the utility of the iPhos toolkit on the data of human metastatic lung cancer cells (CL1-5).

Conclusions: In the comparison study of the control group of CL1-5 cell lysates and the treatment group of dasatinib-treated CL1-5 cell lysates, we demonstrated the applicability of the iPhos toolkit and reported the experimental results based on the iPhos-facilitated phosphoproteome investigation. And further, we also compared the strategy with pure DDA-based LC-MS/MS phosphoproteome investigation. The results of iPhos-facilitated targeted LC-MS/MS analysis convey more thorough and confident phosphopeptide identification than the results of pure DDA-based analysis.
\end{abstract}

\footnotetext{
* Correspondence: liaopc@mail.ncku.edu.tw; wessonwu@mail.ncku.edu.tw

${ }^{1}$ Department of Electrical Engineering, National Cheng Kung University,

Tainan, Taiwan

${ }^{2}$ Department of Environmental and Occupational Health, National Cheng

Kung University, Tainan, Taiwan

Full list of author information is available at the end of the article
}

(c) 2014 Yang et al.; licensee BioMed Central Ltd. This is an Open Access article distributed under the terms of the Creative Commons 


\section{Background}

Phosphorylation is a crucial protein post-translational modification (PTM) in many biological processes [1]. And many human diseases, such as cancer and the Alzheimer's disease, are discovered to be triggered by the dysregulation of phosphorylation and dephosphorylation $[2,3]$. In eukaryotes, protein kinases catalyze the addition of phosphate groups to the side chains of hydroxyl-containing amino acids (serine, threonine and tyrosine) $[2,3]$. In particular, over 45 protein tyrosine kinases have been implicated in the pathogenesis of human cancers [4]. And nowadays tyrosine-phosphorylated (pTyr) proteins are specific targets for the development of potential biomarkers in prognosis, diagnosis and prediction of drug responses [5]. But the low stoichiometry of phosphorylated proteins is still a significant challenge for identifying them [6].

In the past, protein phosphorylation is detected by antibodies recognizing specific phosphorylated epitopes and/or by the use of ${ }^{32} \mathrm{P}$ labelled $\gamma$ ATP to incorporate labelled phosphorylation into proteins. These labourintensive procedures are prone to false phosphorylation site assignment and hence are not suitable for comprehensive analysis [7]. With the development of liquid chromatography mass spectrometry (LC-MS) technology coupled with refined protein enrichment methods, such as immobilized metal affinity chromatography with $\mathrm{Fe}$ (III) or $\mathrm{Ga}(\mathrm{III})[8,9]$, metal oxide affinity chromatography with $\mathrm{TiO}_{2}$ or $\mathrm{ZrO}_{2}[10,11]$ or phosphoramidate chemistry [12], it is becoming more and more available for large scale phosphoproteome investigation $[13,14]$.

For many years, collision induced dissociation (CID) is the major core method for tandem MS scan of peptides and proteins with/without PTMs. Other fragmentation methods such as electron transfer dissociation (ETC) were also proposed to identify phosphorylated proteins but are generally not efficient to be used in the analysis of lowly-abundant pTyr peptides [15]. When undergoing CID, neutral loss of $98 \mathrm{Da}\left(\mathrm{H}_{3} \mathrm{PO}_{4}\right)$ for peptides with phosphorylated-serine (pSer) and phosphorylated-threonine (pThr) would occur and impede the identification of these peptides in data dependent MS/MS [16]. Instead, neutral loss scan, such as $\mathrm{MS}_{3}$ and MultiStage Activation, that imposes additional activation events on the neutral loss peaks can be utilized to detect pSer and pThr peptides $[17,18]$. But tyrosine phosphorylation is largely exempt from the $\beta$-eliminated neutral loss of 98 $\mathrm{Da}$ and are not suitable for these type of neutral loss scan analysis [16]. The identification of pTyr proteins are mainly through the data-dependent acquisition (DDA)-based LC-MS/MS, which adopts some userdefined criteria to serially select the top intense ions in a survey MS scan of all precursor ions for subsequent CID fragmentation and generates the corresponding product ion spectra for protein database searching or manual interpretation. An alternative for detecting tyrosine phosphorylated peptides is to detect selectively the signal at $\mathrm{m} / \mathrm{z}$ of 216.043 (the immonium ion) $[16,19,20]$. Though the neutral loss scan experiments, pure DDAanalysis and selective detection of immoniun ion work well in semi-complex mixtures, in real complex samples such as cell lysates these types of analysis tend to fail to identify most of the phosphopeptides due to the low abundance of phosphorylated peptides and the suppression effect in the presence of those non-phosphorylated peptides $[21,22]$.

To overcome this obstacle, there is a proposed alternative in which biologist combine the alkaline phosphatase (AP) treatment to facilitate a reference-based signal data mining for phosphoproteome analysis [23]. In this additional AP treatment after standard phosphopeptide enrichment procedures such as $\mathrm{TiO}_{2}$ microcolumns, researchers further focused on the signals with mass shift of some multiples of $79.966 \mathrm{Da}\left(\mathrm{PO}_{3}{ }^{3-}\right)$ detected by comparing the phosphopeptide signals and the corresponding dephosphorylated peptide signals. This has been shown to provide more confident and comprehensive phosphoproteome investigation with lower false positive rate [7,24-26]. And this AP-treatment alternative approach targeting on those signals with mass shift are also shown to provide better discrimination of $\mathrm{pTyr}$ peptides in the lung cancer cell lysates [27]. While the overall process, including tyrosine-specific antibody treatment, AP treatment, mass shift calculation and label-free quantification, is applicable, the key integration along the pipeline was mostly done by tedious manual work or private in-house software. To promote and facilitate this comprehensive data mining-based phosphoproteome investigation, developing the fully facilitating toolkit is urgent.

To facilitate the overall workflow of the AP-assisted comprehensive phosphoproteome investigation, we developed the software package, iPhos. iPhos is a collection of signal data mining and analysis facilitating tools to streamline the workflow for routine phosphoproteome characterization. The iPhos toolkit includes one assister and three modules. iPhos Module-1 is used for signal processing of the LC-MS signal peaks extracted by peak extraction computational tools to mine out signals with mass shift between the original and dephosphorylated samples. In addition to iPhos Module-1, we further design the iPhos Peak Extraction Assister to help users automate the batch mode msInspect-based peak extraction procedure [28]. iPhos Module- 2 provides customized $\mathrm{m} / \mathrm{z}$ inclusion lists with peak retention time windows for subsequent targeted LC-MS/MS experiments. iPhos Module-3 is designed to aid the pattern-based label-free quantification. Since most of the pattern-based label-free tools did not link the 
information of peptide identification to the quantification results [29], we developed iPhos Module-3 to connect specific phosphopeptide identifications with quantification results generated by pattern-based label-free quantification tools. In addition, iPhos Module-3 can help researchers filter out those peptides with proper phosphorylation site assignment. The iPhos toolkit aims to facilitate the APassisted and data mining-based comprehensive phosphosproteome investigation and quantification. The utility of the iPhos toolkit was evaluated through the analysis of the differential expression levels of pTyr proteins between metastatic lung cancer cells with tyrosine kinase inhibitortreatment and cells without treatment. We reported the results of the tyrosine phosphosproteome investigation facilitated by iPhos and showed the improved pTyr peptide identification over the results generated by the method of pure DDA LC-MS/MS phosphoproteome analysis. The iPhos toolkit is available online at http://cosbi3. ee.ncku.edu.tw/iPhos/.

\section{Implementation}

The developed iPhos software toolkit was written in Python 2.6 and the web service was built using the PHP CodeIgniter framework. The overall experiment workflow of conducting comparison studies based on the AP-assisted phosphoproteome investigation was proposed by various researchers [22-24,26] and was summarized by Wu et al. [27] (Figure 1). We developed the key integrative tools, collectively called iPhos, to facilitate the workflow. These key integrative tools are described as follows.

\section{Raw data conversion}

The raw files for the AP-treated and untreated samples generated by mass spectrometers should first be converted into the open file representation format of mzXML, which is now a routine job for analysing MS data. It can be done by suitable programs developed by the Seattle Proteome Centre (SPC) [30]. For example, the ReAdW program can be used for raw files from the LTQ-Orbitrap Velos hybrid mass spectrometers. Or users can use the recently updated tool MSConvert provided in the ProteoWizard project [31], by which a GUI interface was constructed for Microsoft Windows users.

\section{iPhos peak extraction assister (iPhos PEA)}

To perform the signal data mining for extracting phosphopeptide signal, we need first to extract the peak signals from the raw experimental LC-MS data. LC-MS data provides 2-dimensional aspect of the proteome under investigation in retention time and $\mathrm{m} / \mathrm{z}$ values and the signals forming peaks in this 2-dimensional map represents the potential peptide signals $[28,32]$. Various algorithms and tools, such as VIPER [33], SuperHirn
[34], Peplist [35], OpenMS [36], ICPD [32], and msInspect [28], have been developed to extract the peak signals. In our previous works, we have demonstrated the use of msInspect in the phosphoproteome investigation [27]. Therefore in the development of the iPhos toolkit, we choose msInspect as the default tool for peak extraction, but users are still freely to select any peak extraction software to generate the peak lists. msInspect acquires peak information after peak detection, monoisotopic peak determination and the calculation of original masses. But msInspect requires the user to allocate proper system memory for the java-based application. Further, batch mode for peak extraction is not possible. Hence in the iPhos toolkit, we developed a peak extraction assister that automate the memory allocation and batch mode peak extraction using the default peak extraction tool msInspect. In this peak extraction assister, users can specify the directory where the mzXML files are located and the peak extraction will be automatically completed by the assister (Figure 1-a).

\section{iPhos Module-1: phosphopeptide signal extraction}

iPhos module- 1 uses the extracted peak information to find the potential phosphopeptide signals according to mass shift of some multiples of -79.966 Da upon dephosphorylation introduced by the AP treatment (Figure 1-b). Users are freely to select any peak extraction software as long as they provide the header information of the peak list files that is to be used in iPhos Module-1. Up to six pairs of replicas with and without AP treatment, respectively, can be processed by iPhos Module- 1 . To avoid the inclusion of noise peak signals, users can set the appropriate cut-off values on the extracted peaks. The intensity threshold can be decided with the aid of the visual charts generated in this module. The cut-off value $\mathrm{N}$ can be determined using the greatest slope change in the output chart to select only the top $\mathrm{N}$ peaks of phosphorylated and dephosphorylated peptides. Finally, users should specify the mass tolerance and retention time window used for signal mass shift mining. In addition, users can also custom the quantity of the mass shift, making iPhos vulnerable to the investigation of other PTMs.

\section{iPhos Module-2: inclusion list generation for targeted LS- MS/MS}

iPhos module- 2 was designed so that users can customize the $\mathrm{m} / \mathrm{z}$ inclusion lists for the downstream targeted LCMS/MS analysis (Figure 1-c). By setting the user-defined parameters on peptide elution times, users can generate equally-divided inclusion list segments of phosphopeptide candidates with their retention time windows that best suit the downstream MS/MS spectrometer [27]. With the limits of mass spectrometers, duplicated $\mathrm{m} / \mathrm{z}$ values resulted from different biological replicas will be eliminated based on the 


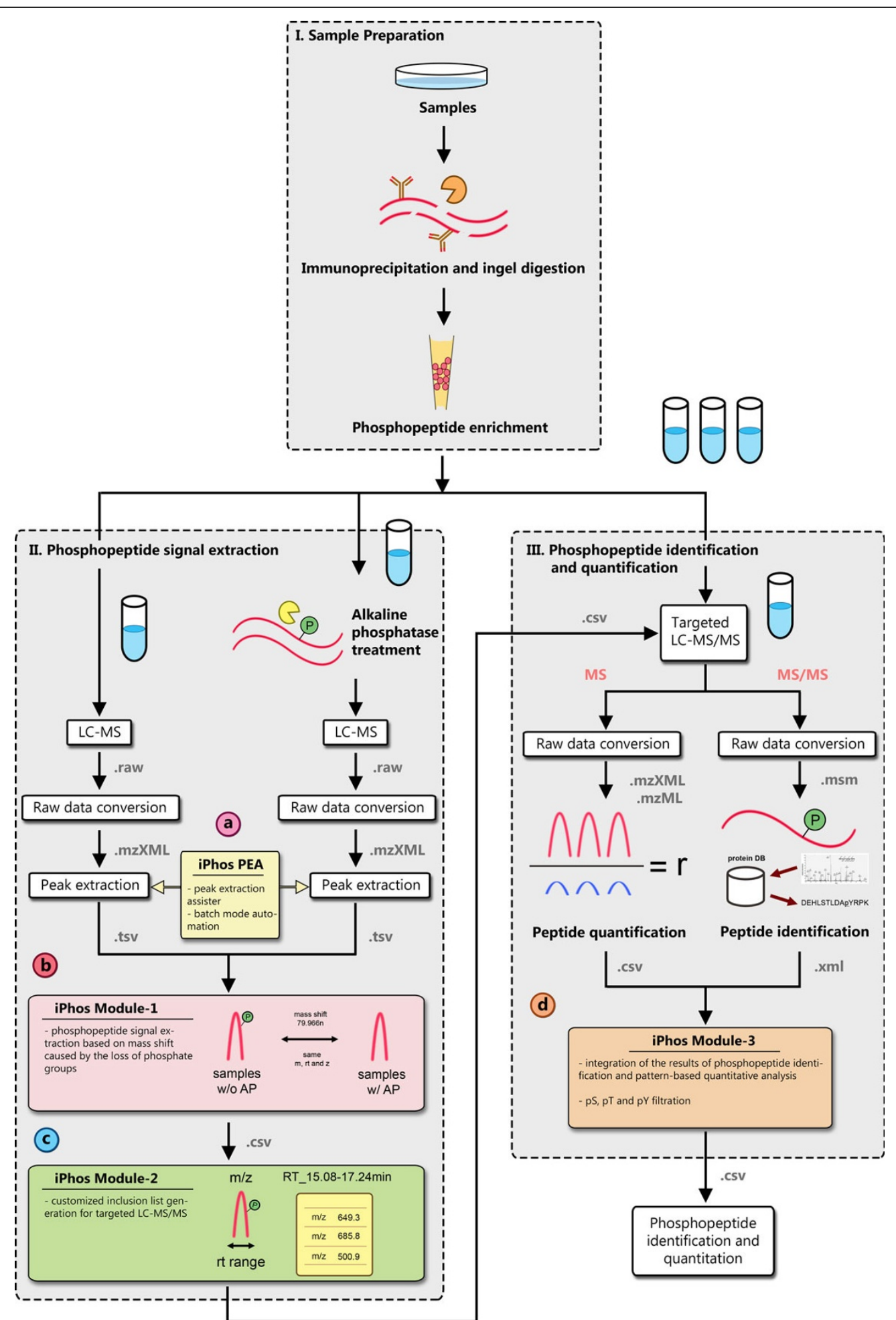

Figure 1 Overall workflow for the iPhos-facilitated comprehensive phosphoproteome investigation. This figure is the sketch plot of the overall iPhos-facilitated phosphorylated peptide identification and quantification work-flow. The input and output file formats for different modules are shown in grey. The key integrative tools, collectively called iPhos, to facilitate the workflow are highlighted in (a)-(d). (a) iPhos Peak Extraction Assister automates the msInspect-based batch mode peak extraction. (b) iPhos Module-1 processes the LC-MS data for phosphopeptide signal extraction. (c) iPhos Module-2 generates customized inclusion lists for subsequent targeted LS-MS/MS analysis. (d) iPhos Module-3 links the results of phosphopeptide identification with pattern-based label-free quantitative analysis. 
round off decimals of $\mathrm{m} / \mathrm{z}$ values. For comparative experiments, iPhos module-2 can combine signals with mass shifts from samples obtained from different conditions [27].

\section{iPhos Module-3: integration of phosphopeptide identification and pattern-based label-free quantitative analysis}

Since the level of phosphorylation of a protein may be crucial for its function, quantitative information for phosphopeptides is required in the phosphoproteome investigation [17]. While traditional quantification of phosphopreteome is based on labelling methods such as SILAC or iTRAQ [16], the cost-efficient method of label free-quantification method is now a catching-on alternative $[26,27,29]$. Label-free method can be categorized into three different types [29]. The first type is identification-based quantification using spectral counts. This type of is generally not suitable for phosphoproteome quantification because the identification of phosphorylation is based on only single or few peptide identification $[18,24]$. The second type is pattern-based quantification using peak identification and XIC calculation. The third type is hybrid-based quantification using the peptide identification as the starting point to calculate the corresponding XIC area. Among these popular quantification methods, the use of SILAC labelling quantification and the pattern-based label-free quantification rely on only MS information. Most of the commercial and open source LC-MS quantification tools based on these two do not link the peptide identification information with the quantification results, thus lacking the phosphorylation site localization.

To facilitate the phosphorylation site localization for quantification tools based on SILAC labelling or patternbased label-free methods, iPhos Module-3 was designed to integrate the peptide identification from the targeted LCMS/MS analysis with the quantification results of LC-MS experiments (Figure 1-d). The LC-MS quantification can be done by pattern-based label-free quantification software tools such as msInspect [28] and mzMine [37], which first extract all peaks from each MS data and then align them across different runs [38]. Users are required to input the header information of the quantification results that is to be used in iPhos Module-3. Peptide identification should be the search results by major database search engines such as Mascot. In iPhos Module-3, the properties of peaks, such as $\mathrm{m} / \mathrm{z}$, retention time and charge state, are utilized to link the phosphopeptide identification from the targeted LC-MS/MS analyses with the quantification results of the corresponding LC-MS comparative experiments. In addition, in iPhos Module-3 users can specify the phosphorylation type (pSer, pThr, pTyr) in concern to further filter out the intended phosphorylated peptides.
iPhos Module-3 can also facilitate the integration of DDA LC-MS/MS peptide quantification and peptide identification in a similar manner (Figure 2). Moreover, iPhos Module-3 can be extended to support for the catching-on data independent acquisition (DIA) LC-MS methods (Figure 3). In the LC-MS DIA experiment settings, the peptide quantitative information can be acquired for each precursor ion during the low energy scan cycle using standard pattern-based quantification tools [39]. For peptide identification, the composite spectra of $\mathrm{MS}^{\mathrm{E}}$ data obtained in the elevated energy scan cycle can be analysed with the standard database search tools (such as Mascot) developed for DDA analysis [40], either by directly searching the composite spectra [41] or by searching the reconstructed pseudo-MS/MS DDA spectra [42-45]. This can be done by software products such as ProteinLynx Global SERVER (PLGS), DeMux [42], ETISEQ [45] and other similar tools. To facilitate the DIA phosphopeptide identification, iPhos Module-3 connects the peptide identification results from any of these database search methods with the standard MS peptide quantification results from pattern-based quantification software. And iPhos Module-3 further helps extract those phosphopeptides of interest with Mascot scores above the user-defined threshold.

\section{Demonstration dataset \\ Cell lysate preparation}

The human lung adenocarcinoma cells CL1-5 cells selected by the in-vitro invasion assay were kindly provided by Dr. Pan-Chyr Yang (Academia Sinica, Taipei, Taiwan) and cultured in the RPMI-1640 medium (Gibco, Gaithersburg, MD) supplemented with 10\% fetal bovine serum (Gibco), penicillin and streptomycin (Thermo Scientific, Logan, Utah). Cells were incubated at $37^{\circ} \mathrm{C}$ containing $5 \% \mathrm{CO}_{2}$. Dasatinib (Santa Cruz Biotechnology, Santa Cruz, CA) was prepared at $0.1 \mathrm{mM}$ in a DMSO vehicle (Sigma-Aldrich, St. Louis, MO). When achieving 70-80\% confluence, parts of the cells were treated with a final concentration of $0.1 \mu \mathrm{M}$ dasatinib in a $0.1 \%$ DMSO vehicle for 48 hours. The control and dasatinib-treated CL1-5 cells were centrifuged at $800 \times \mathrm{g}$ for $10 \mathrm{~min}$. Both cell pellets were suspended in PBS buffer and subsequently lysed by $2 \mathrm{ml}$ lysis buffer $(50 \mathrm{mM}$ Tris- $\mathrm{HCl}, 150 \mathrm{mM} \mathrm{NaCl}, 1 \% \mathrm{NP}-40,0.5 \%$ sodium deoxycholate, $0.1 \%$ SDS, $1 \times$ protease inhibitor cocktail (Roche Applied Science, Indianapolis, IN), and phosphatase inhibitor mixture $(2 \mathrm{mM}$ sodium orthovanadate, $5 \mathrm{mM}$ sodium fluoride, and $10 \beta$-glycerolphosphate)). After centrifuged at $12,000 \times \mathrm{g}$ for one hour, the supernatant was collected. Some of the supernatant was then extracted for protein concentration estimation by using a bicinchoninic acid (BCA) protein assay (Pierce, Rockford, IL). 


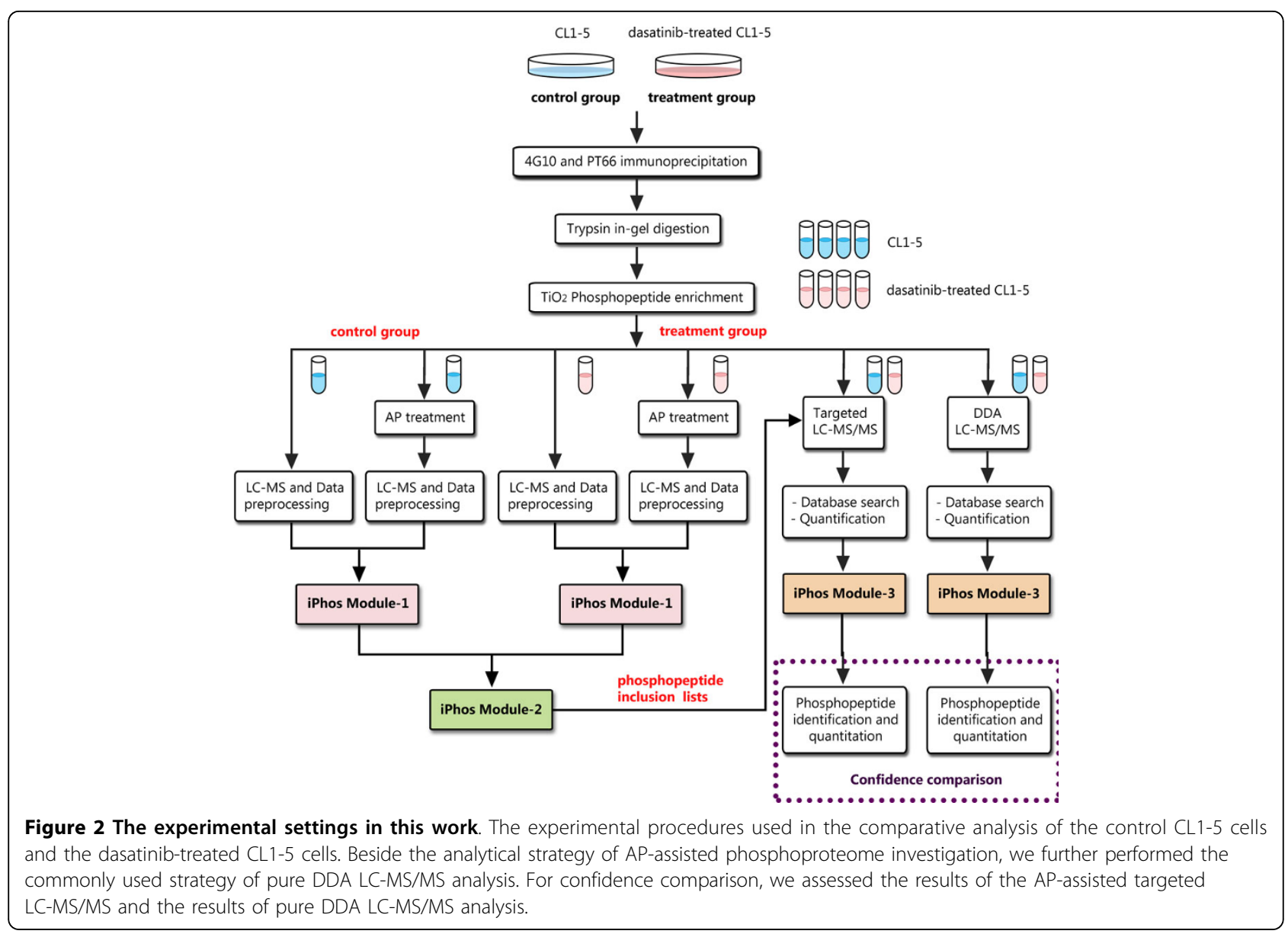

\section{Immunoprecipitation and in-gel digestion}

$1.4 \mathrm{mg}$ of proteins were resuspended in $600 \mu \mathrm{l}$ of lysis buffer and then reacted with $100 \mu \mathrm{l}$ of agarose-immobilized pTyr-specific antibody 4G10 (Millipore, Temecula, CA) and PT66 (Sigma-Aldrich). After the antibody treatment, samples were washed three times with $1 \mathrm{ml}$ radioimmunoprecipitation assay (RIPA) buffer and eluted using $80 \mu \mathrm{l} 4 \times$ sample buffer (Invitrogen, La Jolla, CA) supplemented with $400 \mathrm{mM}$ dithiothreitol (DTT). To concentrate the eluted proteins, SDS-PAGE composed of a $5 \%$ acrylamide stacking gel and a $20 \%$ acrylamide separating gel was used. After electrophoresis at $50 \mathrm{~V}$ for $30 \mathrm{~min}$, the gel was then stained with Coomassie blue for one hour. The protein band was passed through a destaining solution $(\mathrm{H} 2 \mathrm{O} / \mathrm{methanol} /$ acetic acid in a ratio of 50/40/10 (v/v/v)) and excised in pieces of $1 \mathrm{~mm}^{3}$, which were then subjected to in-gel digestion.

The destained gel pieces were washed twice with solution of $50 \%(\mathrm{v} / \mathrm{v})$ acetonitrile $(\mathrm{ACN}) / 25 \mathrm{mM}$ ammonium bicarbonate. The spots were reduced with $10 \mathrm{mM}$ DTT for one hour at $56^{\circ} \mathrm{C}$ and alkylated with $55 \mathrm{mM}$ iodoacetamide for $45 \mathrm{~min}$ in the dark at room temperature. Subsequent digestion was performed by incubating the samples with $0.1 \mu \mathrm{g}$ of TPCK-treated modified porcine trypsin (Promega, Madison, WI) at $37^{\circ} \mathrm{C}$ overnight. The extracts of the pieces were prepared using $20 \mu \mathrm{l}$ of $50 \%$ CAN / 5\% formic acid; these extracts were combined with the supernatant containing the tryptic peptides. The samples were then dried using a centrifugal evaporation instrument (Vacufuge, Eppendorf, Hamburg, Germany).

\section{Phosphopeptide enrichment by $\mathrm{TiO}_{2}$ pipette tips and AP treatment}

The tryptic peptides were suspended with $100 \mu \mathrm{l}$ solution A (0.1\% Trifluoroacetic acid (TFA), $80 \%$ ACN with $300 \mathrm{mg} / \mathrm{ml}$ lactic acid) and loaded onto the $\mathrm{TiO}_{2}$ pipette tip $(200 \mu \mathrm{l} / 3 \mathrm{mg}$, GL science, Torrance, CA) for phosphopeptide binding. After centrifuge, we repeated the binding step twice. The wash step was performed with solution A and solution B (0.1\% TFA, $80 \% \mathrm{ACN})$ successively. Then $50 \mu \mathrm{l} 0.5 \%$ ammonium hydroxide was added to the tips for elution. A quarter of the eluted samples were further treated with alkaline phosphatase (AP). This quarter of the samples for AP treatment was adjusted with dephosphorylation buffer (Roche) to $\mathrm{pH}$ 8-9 and then incubated with $1 \mathrm{U}$ of AP (Roche) at $37^{\circ} \mathrm{C}$ 


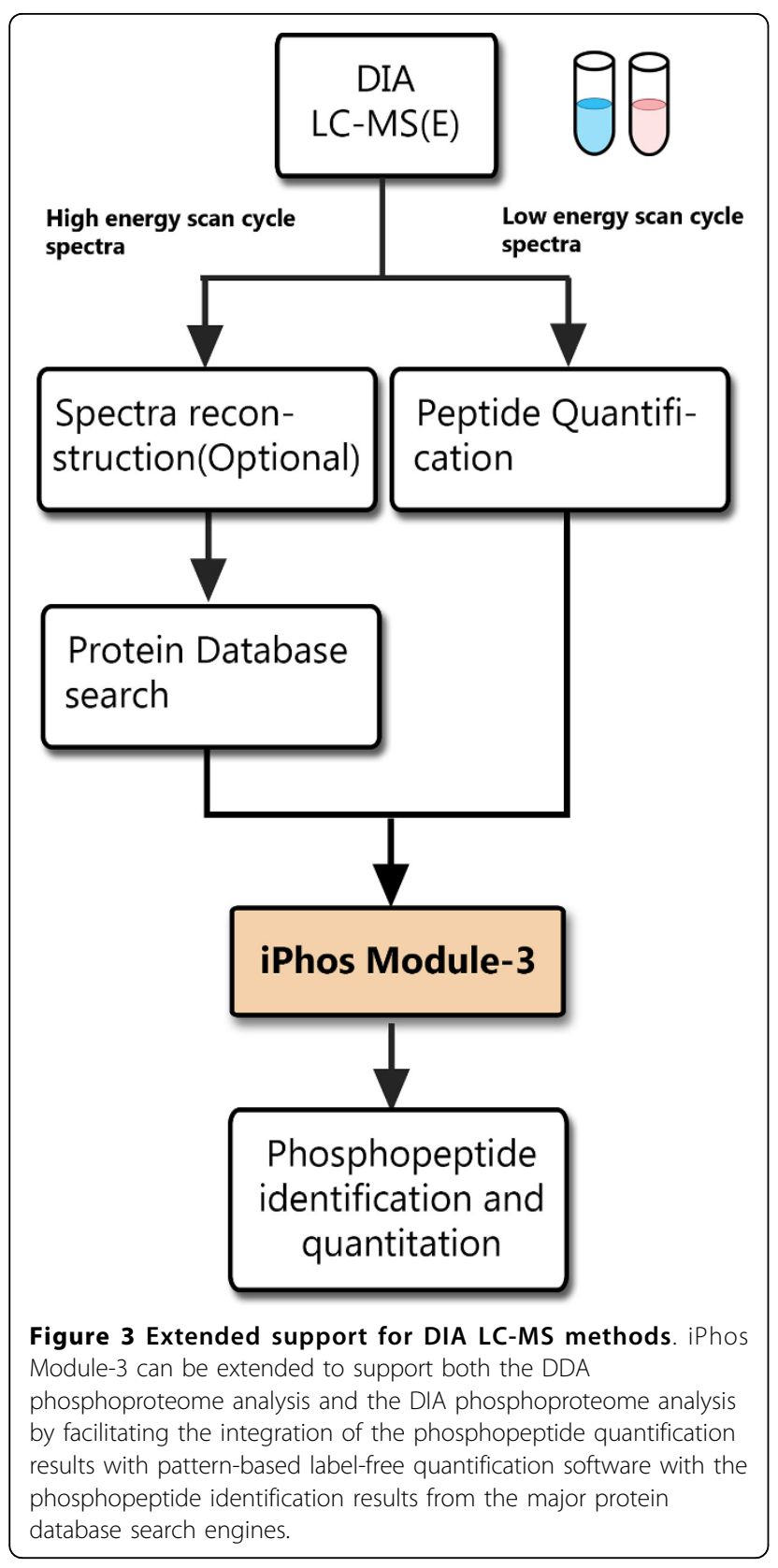

for $2 \mathrm{~h}$. After that, $2.5 \%$ TFA were added to all the eluted samples and the samples were desalted by $\mathrm{C} 18$ pipet tips (Varian, Lake Forest, CA). The eluate was evaporated and suspended with $10 \mu \mathrm{l}$ of $5 \% \mathrm{ACN}$ in $0.1 \%$ TFA solution before nanoLC-MS analysis. For generating the targeted phosphopeptide signals in this study, we categorized four experimental groups: CL1-5, CL1-5 with AP treatment, dasatinib-treated CL1-5, and dasatinib-treated CL1-5 with AP treatment.

\section{NanoLC-MS analysis}

The samples were analysed by full-scan LC-MS on a nano-ACQUITY system (Waters, Milford, MA) coupled with an LTQ-Orbitrap Velos hybrid mass spectrometer (Thermo Scientific) which is equipped with a PicoView nanospray interface (New Objective). Peptide mixtures were loaded onto a $75-\mu \mathrm{m} \times 250$-mm nano-ACQUITY UPLC BEH130 column packed with C18 resin (Waters) and were separated at a flow rate of $300 \mathrm{nl} / \mathrm{min}$ using a linear gradient of $5 \%$ to $40 \%$ solvent B ( $95 \%$ acetonitrile with $0.1 \%$ formic acid) for $60 \mathrm{~min}$, followed by a sharp increase to $85 \%$ solvent $\mathrm{B}$ at $1 \mathrm{~min}$ and then a holding period at $85 \%$ solvent B for another $10 \mathrm{~min}$. Solvent A was $0.1 \%$ formic acid in water. The effluent from the HPLC column was directly electrosprayed into the mass spectrometer. The LTQ-Orbitrap Velos instrument was operated in full scan MS acquisition mode. Full scan MS spectra (m/z 300-2000) were acquired in the Orbitrap analyser with resolution set at 60,000 (all Orbitrap system resolution values are given at $\mathrm{m} / \mathrm{z} 400$ ). The standard mass spectrometric conditions for all experiments were as the following: spray voltage, $1.8 \mathrm{kV}$; no sheath and auxiliary gas flow; heated capillary temperature, $200{ }^{\circ} \mathrm{C}$; predictive automatic gain control (AGC) enabled; and an S-lens RF level of $50 \%$. The instrument was controlled by Tune 2.6.0 and Xcalibur 2.1 software.

\section{Targeted LC-MS/MS analysis and database search}

The $\mathrm{m} / \mathrm{z}$ values with retention time windows of possible pTyr peptide signals were obtained from iPhos Module2. We further divided the remaining half of the samples into two portions. The inclusion lists on the same LTQOrbitrap Velos hybrid mass spectrometer triggered MS/ MS analysis once these $\mathrm{m} / \mathrm{z}$ values were captured on one portion of the pooled control and treated samples. The other portion was subjected to data-dependent acquisition (DDA) as a comparison with the targeted LC-MS/MS analysis. Each scan of the mass spectrometer contained one full scan mass spectrum $(\mathrm{m} / \mathrm{z}$ 300-2000) followed by 20 tandem mass spectra with the collision energy set at 35\%. The targeted LC-MS/MS mode was devised that ten most intense ions from the inclusion list were used for LC-MS/MS analysis. In the targeted MS/MS mode, if there was no signal matching the inclusion list in full scan, the DDA mode was used. The method of multi-stage activation was applied and the neutral losses were set as 97.98, 79.97, 48.99, 39.98, 32.66 , and 26.66 .

All MS raw data were processed by Raw2MSM and searched against the IPI human database (Version 3.87) using an in-house Mascot server (Version 2.2, Matrix Science Ltd., London, UK) with a confidence level of $95 \%$. Up to two missed cleavages were allowed for the trypsin digestion simulation. We allowed the variable modifications of Carboxyamidomethylation on Cys, deamidation on Asp and Glu, oxidation on Met, and phosphorylation on Ser, Thr, and Tyr. Mass deviations for precursor ions and fragment ions were all set to $1 \mathrm{Da}$. A false discovery 
rate of $\leq 5 \%$ was set based on the calculation by the Mascot-integrated decoy database search.

\section{Results and discussion}

CL1-5 cell lysate phosphoproteome investigation assisted by iPhos

To demonstrate the utility of the iPhos toolkit in a large-scale quantitative tyrosine phosphoproteomics study, two enriched pTyr protein mixtures were prepared from the control and dasatinib-treated CL1-5 cells. It has been shown that several pTyr proteins involved in Src kinase regulation have different expression levels in the human metastatic lung cancer cell line CL1-5 compared with the less invasive sub-population of CL1-0 [27]. And dasatinib is an anti-tumour drug that inhibits Src family tyrosine kinases and is frequently used to evaluate changes in pTyr protein expression levels before and after treatment [46,47]. pTyr proteins were immunoprecipitated using a combination of two pTyr specific antibodies, 4G10 and PT66. To form a comparison study, we use the CL1-5 cells as the control group and dasatinib-treated CL1-5 cells as the treatment group. Half of both samples were further divided into two portions and one of this sub-portion was treated with alkaline phosphatase. The other half of both samples was also divided into two portions: one portion used for targeted LC-MS/MS analysis and the other portion used for DDA LC-MS/MS analysis. We scattered each part into three technical replicas to measure reproducibility. The overall datasets and settings of the comparison study are shown in Figure 2.

We set the following parameters in iPhos Module-1: top 3000 peaks cut-off value in phosphorylated and dephosphorylated runs; 79.966 Da mass shift; 0.05 Da mass tolerance; 5 min peak retention time shift tolerance; up to 5 phosphate groups modified; charge state at least 2 . Then we loaded the potential phosphopeptide signals mined out from both the control and treatment group by iPhos Module-1 into iPhos Module-2. In iPhos Module-2, we set the time range to be 10 to $60 \mathrm{~min}$ according to the exact peak elution time in the gradient of solvent $\mathrm{B}$ and generated the inclusion lists by separating these potential pTyr peptides signals into $10 \mathrm{seg}$ ments with 1.5 min extra retention time tolerance in each segment. The round-off decimal was set to be 2 . Overall, 262 unique signals with specific retention time window was found and input as the "inclusion list" in the LTQ-Orbitrap Velos mass spectrometer for targeted LC-MS/MS analysis. These 262 signals were extracted from about 19,000 monoisotopic peaks presented in all LC-MS runs, including the original samples and the dephosphorylated samples (Figure 4).

Then we used iPhos module- 3 to combine the quantification results from Progenesis (Nonlinear Dynamics,
Newcastle upon Tyne, UK) and the pTyr peptide identification results from the Mascot search engine. The parameters used in iPhos Module- 3 was set as the following: Mascot score higher than 25; $0.2 \mathrm{Da} \mathrm{m} / \mathrm{z}$ tolerance; $2 \mathrm{~min}$ retention time tolerance; pTyr peptide modification. Beside the spiked-in internal standard peptides (MSYNCCSGNFSSR), a total of 125 pTyr peptides are identified and quantifiable in the control and treatment group (See Additional File 1). And there are 18 differentially expressed pTyr peptides (using the ANOVA test with $\alpha=0.05$, shown in Table 1 ).

\section{Demonstration example of AP-assisted phosphopeptide identification}

The central dogma of the iPhos-facilitated phosphoproteome investigation is based on the loss of phosphate group(s) (-79.966n Da) to extract potential phosphopeptide signals as the inclusion lists for targeted LC-MS/MS analysis. We take a demonstration example from our datasets to show the idea. The partial sequence (DEHLSTLDApYRPK) of the protein named "Similar to ankyrin repeat domain 20A, isoform CRA_a" was identified in all targeted LC-MS/MS runs. After signal data mining of iPhos Module- 1 and Module-2, the original peptide mass of $1623.701 \mathrm{Da}$ (from m/z 812.858, 2+) in the APuntreated run compared with $1543.725 \mathrm{Da}$ (from 772.870, $2+)$ in the AP-treated run reveals a mass shift of -79.9767 $\mathrm{Da}\left(\mathrm{HPO}_{3}\right)$ (Figure $\left.5-\mathrm{a}\right)$. The $\mathrm{m} / \mathrm{z}$ of 812.8579 was rounded to 812.86 and set as the potential peak signal in the inclusion list with retention time window from 21.51 to 28.43 min. After the targeted LC-MS/MS scan, the pTyr peptide sequence of DEHLSTLDAPYRPK was identified and reported by the Mascot search engine with a score of 39 and eluted in the time range of 23.32 to $27.25 \mathrm{~min}$. The MS/MS spectrum of this peptide signal is shown in Figure 5 -b. Notice that the elution time found by the MS/MS analysis coincided with the retention time window provided by iPhos Module- 2 .

\section{Improvement over DDA-based phosphoproteome investigation}

iPhos-facilitated investigation obtains more $p$ Tyr peptide identification and reproducibility

We have divided our samples to separately perform the iPhos-facilitated target LC-MS/MS analysis and DDA LCMS/MS analysis for comparison. These two sub-portions are identically prepared but are subject to different LCMS/MS analysis. We manually collected the identified pTyr peptides in these two analyses. There are 308 and 255 pTyr peptides identified by the Mascot search engine (score $\geq 25$ ) in the targeted LC-MS/MS analysis and DDA LC-MS/MS runs, receptively. A $20.1 \%$ improvement on the number of identified pTyr peptides was obtained from the iPhos-facilitated phosphoproteome investigation. Of 


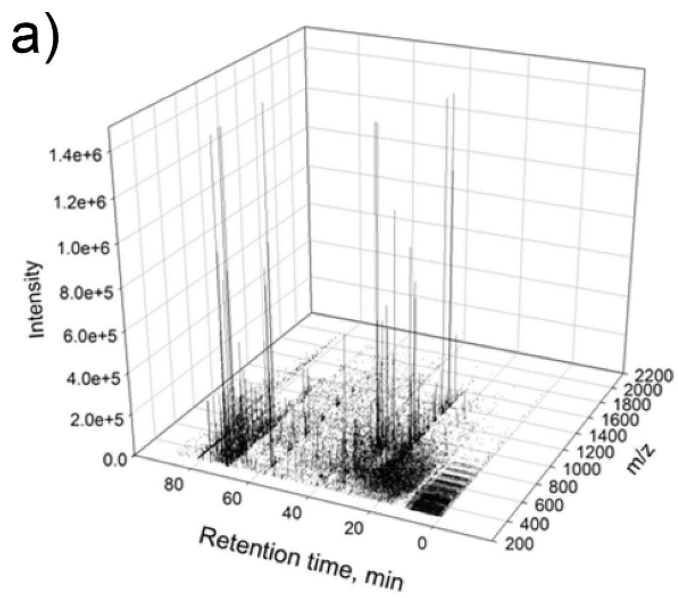

b)

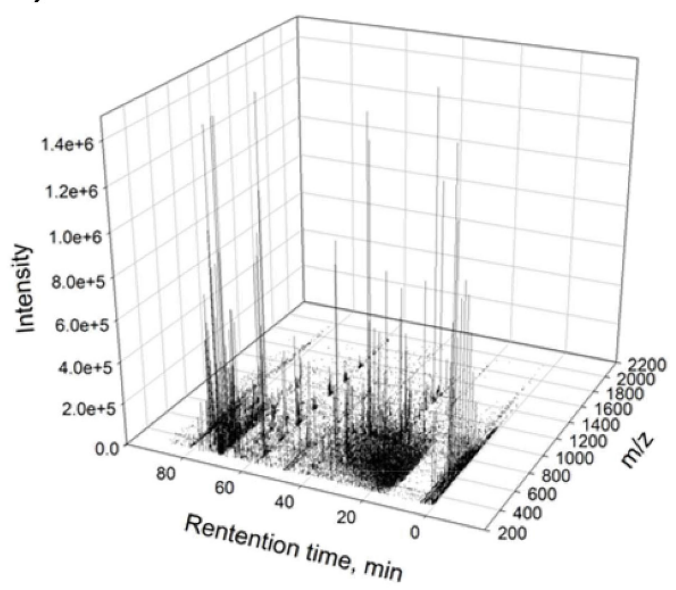

c)

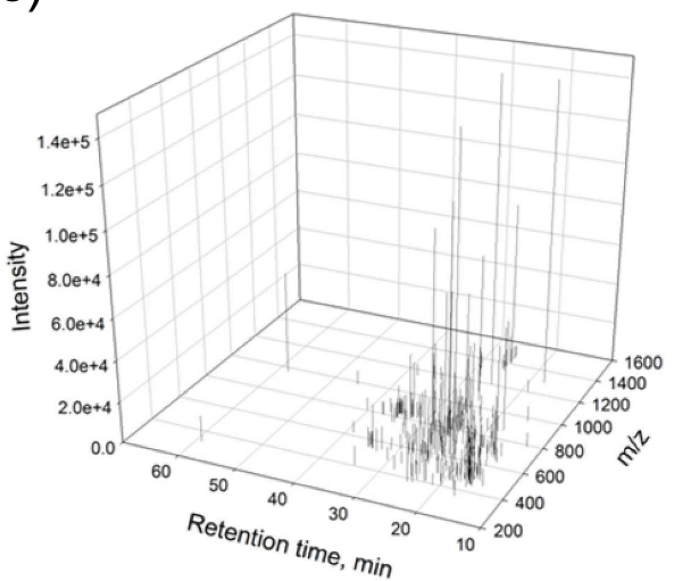

Figure 4 Illustration of phosphopeptide signal data mining. Three-dimensional (retention time, $\mathrm{m} / \mathrm{z}$ and signal intensity) representation of the LC-MS signals before and after the signal data mining of iPhos Module-1. (a) Approximately 19,000 monoisotopic signals are in the tryptic pTyr-enriched protein mixture samples that were not alkaline phosphatase treated. (b) The signals in the tryptic pTyr-enriched protein mixture that has undergone alkaline phosphatase dephosphorylation. (c) iPhos Module-1 extracted 262 possible pTyr signals with mass shifts of some multiples of phosphate group loss. 
Table 1 Eighteen pTyr peptides with differential expression levels between the control and dasatinib-treated CL1-5 cells

\begin{tabular}{|c|c|c|c|c|c|c|c|}
\hline & $\begin{array}{l}\text { IPI } \\
\text { number }\end{array}$ & $\begin{array}{l}\text { Gene } \\
\text { Symbol }\end{array}$ & Protein name & Sequence & $\mathrm{m} / \mathbf{z}$ & $\begin{array}{l}\text { Ratio (control } \\
\text { / treatment) }\end{array}$ & ANOVA \\
\hline 01 & IPI00977158 & CTSF & Cathepsin F & $\mathrm{pT} R T \mathrm{R} \mid \mathrm{p} \mathbf{Y}\lfloor\mathbf{N} T L L R K$ & 826.8552 & infinity & $3.8 \mathrm{E}-4$ \\
\hline 02 & IPI00003384 & CELSR1 & $\begin{array}{l}\text { Cadherin EGF LAG seven-pass G-type } \\
\text { receptor } 1\end{array}$ & DRDANSVITpYQLpTGGNTR & 714.9915 & 8.57 & $1.7 \mathrm{E}-2$ \\
\hline 03 & IPI00908866 & MGAT4A & $\begin{array}{l}\text { Alpha-1,3-mannosyl-glycoprotein 4-beta-N- } \\
\text { acetylglucosaminyl-transferase A }\end{array}$ & NpYSITVpSIVMGIPTVK & 632.9769 & 4.51 & 4.4E-2 \\
\hline 04 & IPI00328929 & ZC3H18 & $\begin{array}{l}\text { Zinc finger } \mathrm{CCCH} \text { domain-containing protein } \\
18\end{array}$ & RpTLSGSGSGSGSSpYSGSSpSR & 701.9993 & 3.33 & $1.0 \mathrm{E}-2$ \\
\hline 05 & IPI01025392 & UBR7 & $\begin{array}{l}\text { Ubiquitin protein ligase E3 component } \\
\text { n-recognin } 7\end{array}$ & PLACSPYECHGSHK & 542.5891 & 2.13 & $3.7 \mathrm{E}-3$ \\
\hline 06 & IPI01014015 & TSPAN19 & Tetraspanin 19 & $\mathrm{p} \mathbf{Y} \subset \mathbf{C G} \mathbf{Q} H N p \mathbf{Y} \mathbf{T} \mathrm{TWIKN}$ KNK & 805.3546 & 1.98 & 2.7E-4 \\
\hline 07 & Pl01009113 & POLR2A & $\begin{array}{l}\text { CDNA FLJ53860, highly similar to DNA- } \\
\text { directed RNA polymerase II largest subunit }\end{array}$ & TLNpTFHpYAGVSAK & 523.5813 & 1.65 & $3.1 \mathrm{E}-2$ \\
\hline 08 & IPI00025468 & SKIL & Ski-like protein & pYLGpTPEEKK & 612.7616 & 1.54 & $3.6 \mathrm{E}-2$ \\
\hline 09 & IPI00981378 & CAMSAP2 & $\begin{array}{l}\text { Calmodulin regulated spectrin-associated } \\
\text { protein family, member } 2\end{array}$ & pTpSSVASGTEpYTGPK & 812.3648 & 0.70 & $2.0 \mathrm{E}-2$ \\
\hline 10 & IPI00977861 & $\mathrm{ABI3BP}$ & ABI family, member 3 (NESH) binding protein & pYKNEpTLALPAESK & 812.3654 & 0.70 & $2.0 \mathrm{E}-2$ \\
\hline 11 & IPI00853187 & LILRB3 & $\begin{array}{l}\text { Leukocyte immunoglobulin-like receptor } \\
\text { subfamily B member } 3\end{array}$ & CGpSQKGpYHHFVLMKE & 970.9699 & 0.67 & $3.1 \mathrm{E}-2$ \\
\hline 12 & $\mathrm{Pl} 00878191$ & DRG1 & $\begin{array}{l}\text { Developmentally-regulated GTP-binding } \\
\text { protein } 1\end{array}$ & $\mathrm{p} \mathbf{Y} L L E K I W D p \mathbf{Y} L K$ & 822.3957 & 0.35 & $2.2 \mathrm{E}-2$ \\
\hline 13 & IPI00738852 & RBM44 & RNA-binding protein 44 & ILGEpYpTSPLpSSK & 767.3362 & 0.34 & $3.2 \mathrm{E}-2$ \\
\hline 14 & IPI00747652 & $\begin{array}{l}\text { CDNA } \\
\text { FL } 60027\end{array}$ & Moderately similar to F-box only protein 25 & RHGpYCpTLGETFNR & 857.407 & 0.17 & $3.1 \mathrm{E}-2$ \\
\hline 15 & IPI00743720 & SLC25A37 & Mitoferrin-1 & pTVpYQLNGLAGpYFK & 857.4067 & 0.17 & $3.1 \mathrm{E}-2$ \\
\hline 16 & IPI00001617 & SOX4 & Transcription factor SOX-4 & SpSAASSPAAGRSPADHRGpYApSLR & 837.7143 & $3.2 \mathrm{E}-2$ & 2.7E-2 \\
\hline 17 & IPI00016594 & C2orf42 & Uncharacterized protein C2orf42 & pSFIQNRDGTpYELFK & 938.9226 & $1.3 \mathrm{E}-2$ & $1.8 \mathrm{E}-2$ \\
\hline 18 & IPI00382739 & $\mathrm{DHX34}$ & $\begin{array}{l}\text { Probable ATP-dependent RNA helicase } \\
\text { DHX34 }\end{array}$ & pYRINLSVLGPApTR & 810.8598 & 0.3 & $2.1 \mathrm{E}-2$ \\
\hline
\end{tabular}

these identified pTyr peptides, 117 pTyr peptides identified in the targeted LC-MS/MS analysis were found in more than one technical replicas and showed reproducibility. In comparison, only $66 \mathrm{pTyr}$ peptides identified in the DDA LC-MS/MS analysis embraced reproducibility. These results are in agreement with observations in other recent studies [48-50].

\section{Confidence evaluation}

Owing to the presence of multiple potential phosphorylation sites in a peptide, the common scoring system in peptide search engines such as Mascot and Sequest do not properly evaluate phosphorylation site assignment [51-53]. Two solutions were proposed. One is to use the Mascot Delta Score (MD-score) for proper evaluation of the phosphorylation site localization [53]. The MD-score method calculates the difference between the top two Mascot ion scores of alternative phosphorylation sites for the identical peptide sequence from Mascot search results. The other evaluation metric is provided by PhosphoRS, a tool to automate confident localization of phosphorylation sites [54]. PhosphoRS provides the individual probability values for each putative phosphorylated site based on the given MS/MS data. We used both the MDscore and PhosphoRS (Proteome Discoverer: version 1.3, Thermo. Scientific) to compare the confidence of the uniquely identified pTyr peptides from the targeted LC-MS/MS analysis and the DDA LC-MS/MS analysis.

As shown in Figure 6-a, the MD-scores of the identified pTyr peptides are higher in the targeted LC-MS/MS analysis $(19.24 \pm 0.19)$ than the MD-scores in DDA LC$\mathrm{MS} / \mathrm{MS}$ analysis $(17.55 \pm 0.68)$. And it is reported that the MD-score should be more than 5 so as to unambiguously determine phosphorylation sites [55,56]. In our experimental results, only $15.18 \%$ (29/191) of the MD-scores in targeted LC-MS/MS analysis are less than 5. In contrast, $24.86 \%$ (47/189) of the MD-score in DDA LC-MS/MS analysis does not reach above the threshold of 5. From the evaluation of the MD-score method, iPhos-facilitated AP treatment phosphoproteome investigation demonstrates the improvement in the phosphorylation site localization confidence over conventional investigation using DDA LC-MS/MS analysis.

The evaluation results using the PhosphoRS are shown in Figure 6-b. In the results of the targeted LC-MS/MS 


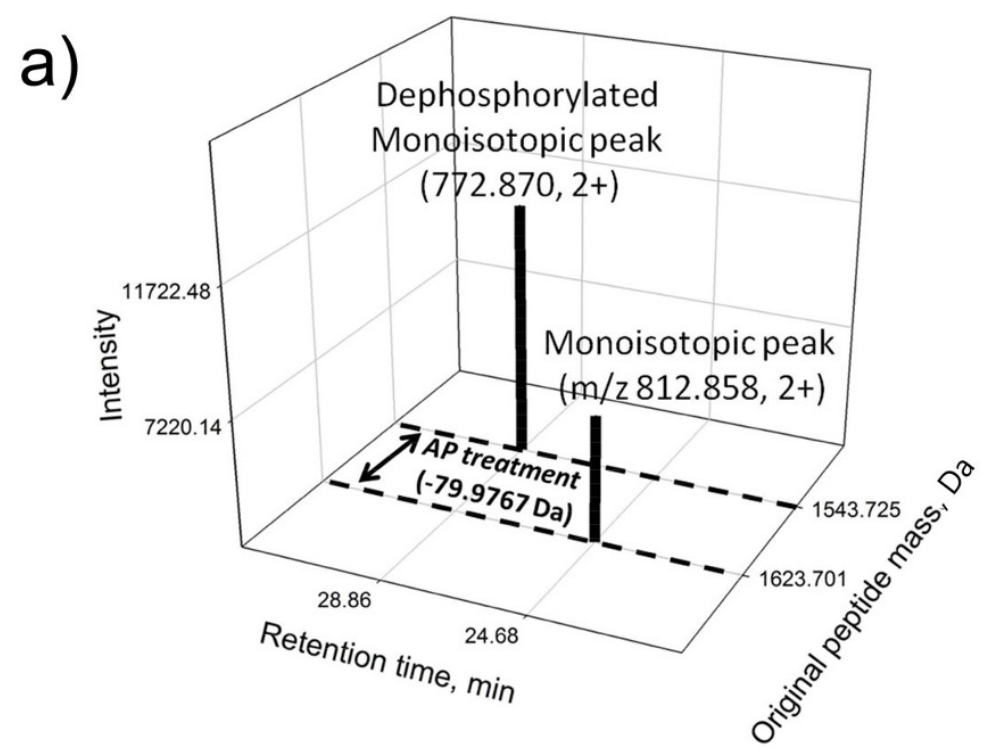

b)

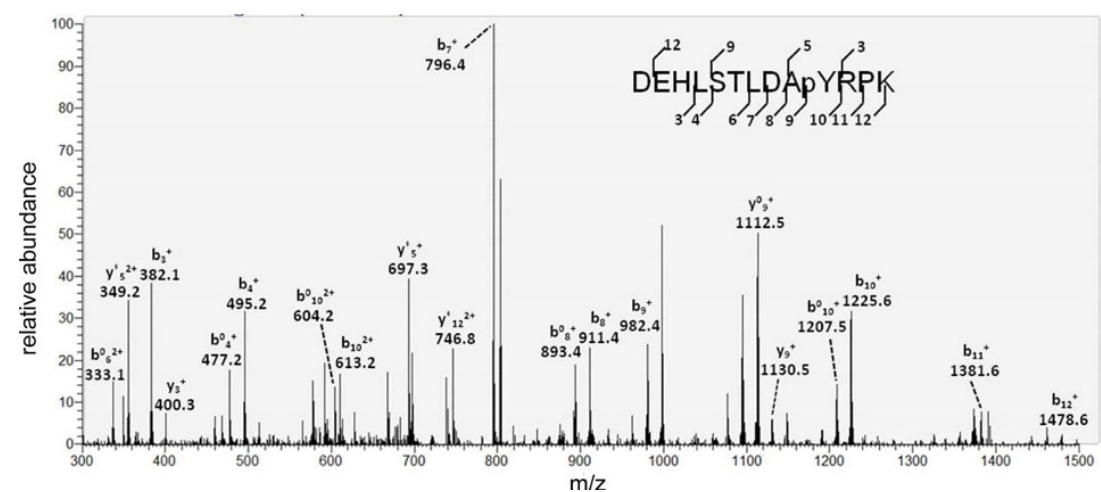

Figure 5 MS/MS spectra of the peptide DEHLSTLDApYRPK. This is a demonstrating example of the iPhos-facilitated phosphopeptide investigation. (a) From iPhos Module-1 and Module-2, the monoisotopic peak with $\mathrm{m} / \mathrm{z}$ value of 812.858 was selected for targeted LC-MS/MS analysis. (b) The MS/MS spectrum of the identified pTyr peptide DEHLSTLDApYRPK. This peptide was identified to be the fragment of the protein "Similar to ankyrin repeat domain 20A, isoform CRA_a" (m/z 812.86, 2+) with a Mascot score of 39.

analysis, 43\% (83/191) phosphorylation localization of the unique pTry peptides are calculated to be confident under the PhosphoRS algorithm. Compared with the results of the targeted LC-MS/MS, only 29\% (55/189) are calculated to be confident in the results of the DDA LC-MS/MS analysis. And among these confident phosphorylation localization, $79.51 \%(66 / 83)$ of them in the targeted LC-MS/MS analysis and 78.18\% (43/55) in the DDA LC-MS/MS analysis are reported to have probability values of correct phosphorylation localization higher than 0.8 , a cut-off value reported to be of acceptable false localization rate. In summary, the evaluation based on the PhosphoRS algorithm shows that iPhos-facilitated AP treatment phosphpoproteome investigation provides more confident phosphorylation localization in comparison with conventional investigation using DDA LC-MS/ MS analysis.

\section{Conclusions}

In this study, we developed the iPhos toolkit to facilitate and to streamline the AP-assisted phosphoproteome investigation and evaluated the usability of iPhos through the comprehensive pTyr phosphoproteome investigation of the CL1-5 cells. And we also demonstrated the overall improvement of the iPhos-facilitated phosphoproteome investigation in the thorough phosphopeptide identification and confident phosphorylation localization over the pure DDA-based method. In conclusion, iPhos facilitates 

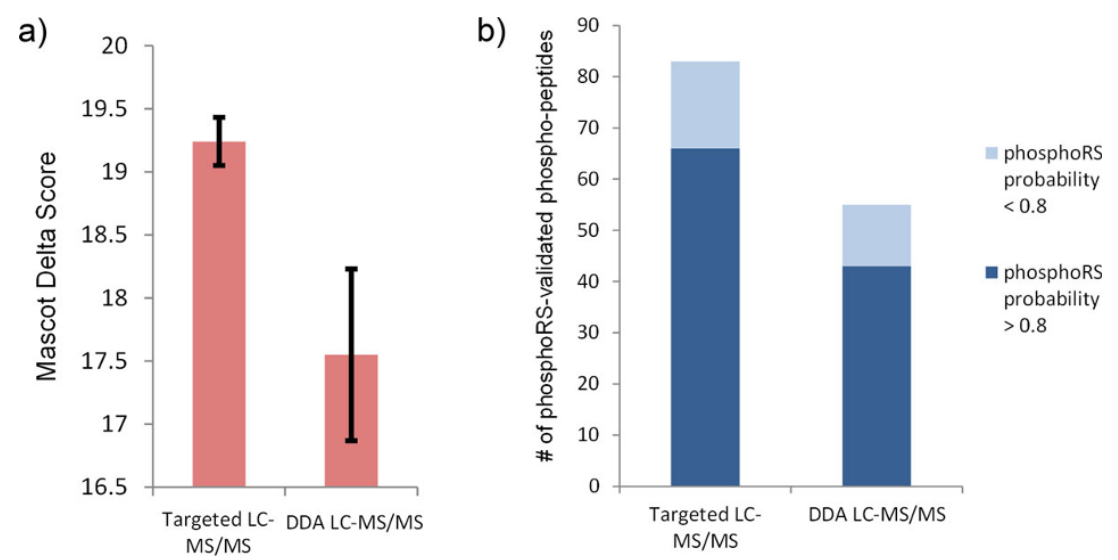

Figure 6 Confidence evaluation of the targeted LC-MS/MS and DDA-LC-MS/MS phosphoproteome investigation. Using the MD-score and PhosphoRS methods, we showed the improvement of the targeted LC-MS/MS-based phosphoproteome investigation over DDA-based analysis. (a) The comparison of the assessment results by Mascot Delta Score (MD-score). The scores of the identified pTyr peptides in the targeted LC-MS/MS runs are higher than those identified pTyr peptides in DDA LC-MS/MS runs (19.24 \pm 0.19 vs. $17.55 \pm 0.68$ ). (b) The comparison of the assessment results by the phosphoRS algorithm in Proteome Discover Software. 83 pTyr peptides (83/191) identified in the targeted LC-MS/MS runs pass the validation. Further, 66 of these validated phosphorylation localization are of high confidence (with probability higher than 0.8). In comparison, only 55 pTyr peptides (55/189) identified in the pure DDA LC-MS/MS runs do. And only 43 of them are of high confidence.

the pipeline of AP-based comprehensive phosphopeptide investigation, allowing for rapid production of vital biological researches on protein phosphorylation with higher confidence.

\section{Availability and requirements \\ Project name: iPhos}

Project home page: http://cosbi3.ee.ncku.edu.tw/ iPhos/

Operating system(s): Windows operating system

Programming language: Python 2.6

Other requirements: Java SDK

License: none

Any restrictions to use by non-academics: none.

\section{Additional material}

Additional file 1: The quantitative information of the pTyr peptides identified by the targeted LC-MS/MS analysis in control and dasatinib-treated CL1-5 cells.

\section{List of abbreviations}

PTM, post-translational modification; pTyr, tyrosine-phosphorylated; LC-MS, liquid chromatography mass spectrometry; pSer, serine-phosphorylated; pThr, threonine-phosphorylated; AP, alkaline phosphatase; DDA, datadependent acquisition; MD-score, Mascot Delta score.

\section{Competing interests}

The authors declare that they have no competing interests.

\section{Authors' contributions}

PCL and HIW conceived the research topic. HTC, CCW and THY developed the iPhos toolkit and set up the iPhos website. SLH generated the datasets and conducted the LC-MS/MS experiments. JLS and THY analysed the experimental data. THY and SLH wrote the manuscript. PCL and WSW provided essential guidance. All authors have read and approved the final manuscript.

\section{Acknowledgements}

This study was supported by National Cheng Kung University and Ministry of Science and Technology of Taiwan (MOST-103-2221-E-006-174-MY2, MOST102-2325-B-006-003, MOST100-2113-M-006-002-MY3 and MOST1032113-M-006-003-MY3)

\section{Declarations}

The publication of this manuscript was funded by Ministry of Science and Technology of Taiwan (MOST100-2113-M-006-002-MY3).

This article has been published as part of BMC Bioinformatics Volume 15 Supplement 16, 2014: Thirteenth International Conference on Bioinformatics (InCoB2014): Bioinformatics. The full contents of the supplement are available online at http://www.biomedcentral.com/bmcbioinformatics/ supplements/15/S16.

\section{Authors' details}

${ }^{1}$ Department of Electrical Engineering, National Cheng Kung University, Tainan, Taiwan. ${ }^{2}$ Department of Environmental and Occupational Health, National Cheng Kung University, Tainan, Taiwan. ${ }^{3}$ Institute of Chemistry, Academia Sinica, Taipei, Taiwan.

Published: 8 December 2014

\section{References}

1. Hunter T: Signaling-2000 and beyond. Cell 2000, 100:113.

2. Pearce $L R$, Komander D, Alessi DR: The nuts and bolts of $A G C$ protein kinases. Nat Rev Mol Cell Biol 2010, 11:9-22.

3. Flirski M, Sobow T: Biochemical Markers and Risk Factors of Alzheimers Disease. Curr Alzheimer Res 2005, 2:47-64.

4. Blume-Jensen $P$, Hunter $T$, et al: Oncogenic kinase signalling. Nature 2001, 411:355-365

5. Lim YP: Mining the tumor phosphoproteome for cancer markers. Clin Cancer Res 2005, 11:3163-3169.

6. Olsen JV, Blagoev B, Gnad F, Macek B, Kumar C, Mortensen P, Mann M: Global, in vivo, and site-specific phosphorylation dynamics in signaling networks. Cell 2006, 127:635-648.

7. Collins MO, Yu L, Campuzano I, Grant SG, Choudhary JS: Phosphoproteomic analysis of the mouse brain cytosol reveals a 
predominance of protein phosphorylation in regions of intrinsic sequence disorder. Mol Cell Proteomics 2008, 7:1331-1348.

8. Porath J: High-performance immobilized-metal-ion affinity chromatography of peptides and proteins. J Chromatogr A 1988, 443:3-11.

9. Posewitz MC, Tempst P: Immobilized gallium (III) affinity chromatography of phosphopeptides. Anal Chem 1999, 71:2883-2892.

10. Pinkse MW, Uitto PM, Hilhorst MJ, Ooms B, Heck AJ: Selective isolation at the femtomole level of phosphopeptides from proteolytic digests using 2D-NanoLC-ESI-MS/MS and titanium oxide precolumns. Anal Chem 2004 76:3935-3943.

11. Kweon HK, Håkansson K: Selective zirconium dioxide-based enrichment of phosphorylated peptides for mass spectrometric analysis. Anal Chem 2006, 78:1743-1749.

12. Bodenmiller $B$, Mueller $L N$, Pedrioli $P G$, Pflieger $D$, Jünger $M A$, Eng JK, Aebersold R, Tao WA: An integrated chemical, mass spectrometric and computational strategy for (quantitative) phosphoproteomics: application to Drosophila melanogaster Kc167 cells. Mol BioSyst 2007, 3:275-286.

13. Gygi SP, Aebersold R: Mass spectrometry and proteomics. Curr Opin Chem Biol 2000, 4:489-494

14. Emadali A, Metrakos PP, Kalantari F, Boutros T, Boismenu D, Chevet E: Proteomic analysis of tyrosine phosphorylation during human liver transplantation. Proteome Sci 2007, 5:1.

15. Syka JE, Coon JJ, Schroeder MJ, Shabanowitz J, Hunt DF: Peptide and protein sequence analysis by electron transfer dissociation mass spectrometry. Proc Natl Acad Sci U A 2004, 101:9528-9533.

16. Johnson $\mathrm{H}$, White FM: Toward quantitative phosphotyrosine profiling in vivo. In Semin Cell Dev Biol. Volume 23. Elsevier; 2012:854-862.

17. Mann M, Ong S-E, Grønborg M, Steen H, Jensen ON, Pandey A: Analysis of protein phosphorylation using mass spectrometry: deciphering the phosphoproteome. Trends Biotechnol 2002, 20:261-268.

18. Rogers $L D$, Foster $L J$ : Phosphoproteomics-finally fulfilling the promise? Mol Biosyst 2009, 5:1122-1129.

19. Collins MO, Yu L, Choudhary JS: Analysis of protein phosphorylation on a proteome-scale. Proteomics 2007, 7:2751-2768.

20. Steen H, Küster B, Fernandez M, Pandey A, Mann M: Detection of tyrosine phosphorylated peptides by precursor ion scanning quadrupole TOF mass spectrometry in positive ion mode. Anal Chem 2001, 73:1440-1448.

21. Wu HY, Tseng VSM, Liao PC: Mining phosphopeptide signals in liquid chromatography-mass spectrometry data for protein phosphorylation analysis. J Proteome Res 2007, 6:1812-1821.

22. Ishihama Y, Wei FY, Aoshima K, Sato T, Kuromitsu J, Oda Y: Enhancement of the efficiency of phosphoproteomic identification by removing phosphates after phosphopeptide enrichment. J Proteome Res 2007, 6:1139-1144

23. Liao P, Leykam J, Andrews P, Gage D, Allison J, et al: An approach to locate phosphorylation sites in a phosphoprotein: mass mapping by combining specific enzymatic degradation with matrix-assisted laser desorption/ionization mass spectrometry. Anal Biochem 1994, 219:9.

24. Imanishi SY, Kochin V, Ferraris SE, de Thonel A, Pallari H-M, Corthals GL, Eriksson JE: Reference-facilitated Phosphoproteomics FAST AND RELIABLE PHOSPHOPEPTIDE VALIDATION BY $\mu$ LC-ESI-Q-TOF MS/MS. Mol Cell Proteomics 2007, 6:1380-1391.

25. Marcantonio M, Trost M, Courcelles M, Desjardins M, Thibault P: Combined Enzymatic and Data Mining Approaches for Comprehensive Phosphoproteome Analyses Application to Cell Signaling Events of Interferon- $\gamma$-Stimulated Macrophages. Mol Cell Proteomics 2008, 7:645-660.

26. Wu HY, Tseng VSM, Chen LC, Chang YC, Ping P, Liao CC, Tsay YG, Yu JS, Liao PC: Combining Alkaline Phosphatase Treatment and Hybrid Linear Ion Trap/Orbitrap High Mass Accuracy Liquid Chromatography- Mass Spectrometry Data for the Efficient and Confident Identification of Protein Phosphorylation. Anal Chem 2009, 81:7778-7787.

27. Wu HY, Tseng VS, Chen LC, Chang HY, Chuang IC, Tsay YG, Liao PC Identification of tyrosine-phosphorylated proteins associated with lung cancer metastasis using label-free quantitative analyses. J Proteome Res 2010, 9:4102-4112.

28. Bellew M, Coram M, Fitzgibbon M, Igra M, Randolph $T$, Wang $P$, May $D$, Eng J, Fang R, Lin C, et al: A suite of algorithms for the comprehensive analysis of complex protein mixtures using high-resolution LC-MS. Bioinformatics 2006, 22:1902-1909.
29. Tsou CC, Tsai CF, Tsui YH, Sudhir PR, Wang YT, Chen YJ, Chen JY, Sung TY, Hsu WL: IDEAL-Q, an automated tool for label-free quantitation analysis using an efficient peptide alignment approach and spectral data validation. Mol Cell Proteomics 2010, 9:131-144.

30. Pedrioli PGA, Eng JK, Hubley R, Vogelzang M, Deutsch EW, Raught B, Pratt $B$, Nilsson E, Angeletti RH, Apweiler R, et al: A common open representation of mass spectrometry data and its application to proteomics research. Nat Biotechnol 2004, 22:1459-1466.

31. Chambers MC, Maclean B, Burke R, Amodei D, Ruderman DL, Neumann $S$, Gatto L, Fischer B, Pratt B, Egertson J, et al: A cross-platform toolkit for mass spectrometry and proteomics. Nat Biotechnol 2012, 30:918-920.

32. Zhang J, Haskins W: ICPD-a new peak detection algorithm for LC/MS. BMC Genomics 2010, 11(Suppl 3):S8.

33. Monroe ME, Tolić N, Jaitly N, Shaw JL, Adkins JN, Smith RD: VIPER: an advanced software package to support high-throughput LC-MS peptide identification. Bioinformatics 2007, 23:2021-2023.

34. Mueller LN, Rinner O, Schmidt A, Letarte S, Bodenmiller B, Brusniak M-Y, Vitek $\mathrm{O}$, Aebersold $\mathrm{R}$, Müller M: SuperHirn-a novel tool for high resolution LC-MS-based peptide/protein profiling. Proteomics 2007, 7:3470-3480.

35. Li X, Eugene $C Y$, Kemp CJ, Zhang $H$, Aebersold R: A software suite for the generation and comparison of peptide arrays from sets of data collected by liquid chromatography-mass spectrometry. Mol Cell Proteomics 2005, 4:1328-1340

36. Sturm M, Bertsch A, Gröpl C, Hildebrandt A, Hussong R, Lange E, Pfeifer N, Schulz-Trieglaff $O$, Zerck A, Reinert $K$, et al: OpenMS-an open-source software framework for mass spectrometry. BMC Bioinformatics 2008, 9:163.

37. Katajamaa M, Miettinen J, Orešič M: MZmine: toolbox for processing and visualization of mass spectrometry based molecular profile data. Bioinformatics 2006, 22:634-636.

38. Radulovic D, Jelveh $S$, Ryu S, Hamilton TG, Foss E, Mao Y, Emili A: Informatics platform for global proteomic profiling and biomarker discovery using liquid chromatography-tandem mass spectrometry. Mol Cell Proteomics 2004, 3:984-997.

39. Blackburn K, Goshe MB: Challenges and strategies for targeted phosphorylation site identification and quantification using mass spectrometry analysis. Brief Funct Genomic Proteomic 2009, 8:90-103.

40. Gillet LC, Navarro P, Tate S, Röst H, Selevsek N, Reiter L, Bonner R, Aebersold R: Targeted data extraction of the MS/MS spectra generated by data-independent acquisition: a new concept for consistent and accurate proteome analysis. Mol Cell Proteomics 2012, 11:0111-016717.

41. Panchaud A, Scherl A, Shaffer SA, von Haller PD, Kulasekara HD, Miller SI, Goodlett DR: Precursor acquisition independent from ion count: how to dive deeper into the proteomics ocean. Anal Chem 2009, 81:6481-6488.

42. Bern M, Finney G, Hoopmann MR, Merrihew G, Toth MJ, MacCoss MJ: Deconvolution of mixture spectra from ion-trap data-independentacquisition tandem mass spectrometry. Anal Chem 2009, 82:833-841.

43. Blackburn K, Mbeunkui F, Mitra SK, Mentzel T, Goshe MB: Improving protein and proteome coverage through data-independent multiplexed peptide fragmentation. J Proteome Res 2010, 9:3621-3637.

44. Geromanos SJ, Vissers JP, Silva JC, Dorschel CA, Li G-Z, Gorenstein MV, Bateman $\mathrm{RH}$, Langridge J: The detection, correlation, and comparison of peptide precursor and product ions from data independent LC-MS with data dependant LC-MS/MS. Proteomics 2009, 9:1683-1695.

45. Wong JW, Schwahn AB, Downard KM: ETISEQ-an algorithm for automated elution time ion sequencing of concurrently fragmented peptides for mass spectrometry-based proteomics. BMC Bioinformatics 2009, 10:244.

46. Gnoni A, Marech I, Silvestris N, Vacca A, Lorusso V: Dasatinib: an antitumour agent via Src inhibition. Curr Drug Targets 2011, 12:563-578.

47. Montero JC, Seoane S, Ocaña A, Pandiella A: Inhibition of SRC family kinases and receptor tyrosine kinases by dasatinib: possible combinations in solid tumors. Clin Cancer Res 2011, 17:5546-5552.

48. Courcelles M, Bridon G, Lemieux S, Thibault P: Occurrence and detection of phosphopeptide isomers in large-scale phosphoproteomics experiments. J Proteome Res 2012, 11:3753-3765.

49. Schmidt A, Gehlenborg N, Bodenmiller B, Mueller LN, Campbell D, Mueller M, Aebersold R, Domon B: An integrated, directed mass spectrometric approach for in-depth characterization of complex peptide mixtures. Mol Cell Proteomics 2008, 7:2138-2150.

50. Haura EB, Müller A, Breitwieser FP, Li J, Grebien F, Colinge J, Bennett KL: Using ITRAQ combined with tandem affinity purification to enhance 
low-abundance proteins associated with somatically mutated EGFR core complexes in lung cancer. J Proteome Res 2010, 10:182-190.

51. Payne SH, Yau M, Smolka MB, Tanner S, Zhou H, Bafna V: Phosphorylationspecific MS/MS scoring for rapid and accurate phosphoproteome analysis. J Proteome Res 2008, 7:3373-3381.

52. Ruttenberg BE, Pisitkun T, Knepper MA, Hoffert JD: PhosphoScore: an open-source phosphorylation site assignment tool for MS $\mathrm{n}$ data. J Proteome Res 2008, 7:3054-3059.

53. Savitski MM, Lemeer S, Boesche M, Lang M, Mathieson T, Bantscheff M, Kuster B: Confident phosphorylation site localization using the Mascot Delta Score. Mol Cell Proteomics 2011, 10:M110-003830.

54. Taus T, Ko"cher T, Pichler P, Paschke C, Schmidt A, Henrich C, Mechtler K: Universal and confident phosphorylation site localization using phosphoRS. J Proteome Res 2011, 10:5354-5362.

55. Sugiyama N, Masuda T, Shinoda K, Nakamura A, Tomita M, Ishihama Y: Phosphopeptide enrichment by aliphatic hydroxy acid-modified metal oxide chromatography for nano-LC-MS/MS in proteomics applications. Mol Cell Proteomics 2007, 6:1103-1109.

56. Wang YT, Tsai CF, Hong TC, Tsou CC, Lin PY, Pan SH, Hong TM, Yang PC, Sung TY, Hsu WL, et al: An informatics-assisted label-free quantitation strategy that depicts phosphoproteomic profiles in lung cancer cell invasion. J Proteome Res 2010, 9:5582-5597.

doi:10.1186/1471-2105-15-S16-S10

Cite this article as: Yang et al.: iPhos: a toolkit to streamline the alkaline phosphatase-assisted comprehensive LC-MS phosphoproteome investigation. BMC Bioinformatics 2014 15(Suppl 16):S10.

\section{Submit your next manuscript to BioMed Central} and take full advantage of:

- Convenient online submission

- Thorough peer review

- No space constraints or color figure charges

- Immediate publication on acceptance

- Inclusion in PubMed, CAS, Scopus and Google Scholar

- Research which is freely available for redistribution

Submit your manuscript at www.biomedcentral.com/submit
Ciomed Central 